\title{
Cellular and Molecular Mechanisms of Spinal Cord Vascularization
}

\author{
Jose Ricardo Vieira ${ }^{1,2 \dagger}$, Bhavin Shah ${ }^{1 \dagger}$ and Carmen Ruiz de Almodovar ${ }^{1,3 *}$ \\ ${ }^{1}$ European Center for Angioscience, Medicine Faculty Mannheim, Heidelberg University, Mannheim, Germany, ${ }^{2}$ Faculty of \\ Biosciences, Heidelberg University, Heidelberg, Germany, ${ }^{3}$ Interdisciplinary Center for Neurosciences, Heidelberg University, \\ Heidelberg, Germany
}

During embryonic central nervous system (CNS) development, the neural and the vascular systems communicate with each other in order to give rise to a fully functional and mature CNS. The initial avascular CNS becomes vascularized by blood vessel sprouting from different vascular plexus in a highly stereotypical and controlled manner. This process is similar across different regions of the CNS. In particular for the developing spinal cord (SC), blood vessel ingression occurs from a perineural vascular plexus during embryonic development. In this review, we provide an updated and comprehensive description of the cellular and molecular mechanisms behind this stereotypical and controlled patterning of blood vessels in the developing embryonic SC, identified using different animal models. We discuss how signals derived from neural progenitors and differentiated neurons guide the SC growing vasculature. Lastly, we provide a perspective of how the molecular mechanisms identified during development could be used to better understand pathological situations.

Keywords: neurovascular, angiogenesis, spinal cord, VEGF, neural progenitors, blood brain barrier, CNS pathology

\section{SPINAL CORD DEVELOPMENT}

The development of the central nervous system (CNS) of vertebrates starts with the formation of the neural tube, an ectoderm-derived embryonic structure that gives rise to the brain and the spinal cord (SC). In mouse, this process starts at E7.5-E8 (embryonic day) and, as the neural tube grows and matures along the anterio-posterior axis, it partitions into the rostral and caudal vesicles. While the rostral vesicles form the prosencephalon, mesencephalon, and the rhombencephalon (together, the brain), the caudal vesicle matures into the SC (Lumsden and Krumlauf, 1996; Purves, 2008). At around E9.5, the neural tube acquires a dorsal-ventral patterning by the controlled secretion of morphogens in a time and concentration dependent manner, establishing 13 different neural progenitor domains (Jessell, 2000; Dessaud et al., 2008). The identity of these progenitors is characterized by a unique expression profile of transcription factors, essential for the specification of the different neuronal subtypes that each domain generates. These individual expression profiles result from the combination of different morphogens: the floor plate and the notochord generate a gradient of Sonic Hedgehog (SHH), the key player for the ventral axis, while the roof plate creates a gradient of Wingless-related integration site (WNTs) family members and bone morphogenic proteins (BMPs), required for dorsal SC patterning. Other important factors, such as fibroblast growth factors (FGFs) and retinoic acid (RA), have been shown to contribute to a correct positioning of the neural 
progenitors (Diez del Corral et al., 2003; Duester, 2008; Diez Del Corral and Morales, 2017). For more details on neural development of the SC, we refer the readers to excellent reviews (Jessell, 2000; Wilson and Maden, 2005; Dessaud et al., 2007, 2008; Sagner and Briscoe, 2019).

Interestingly, as the neural progenitors undergo active proliferation and differentiation, the developing CNS starts to be simultaneously vascularized to meet the increasing demand of nutrients and oxygen. The avascular CNS starts to be vascularized at E8.5-E9.5 in mice by the formation of the perineural vascular plexus (PNVP) from mesodermderived angioblasts that completely covers the entire neural tube (Hogan et al., 2004; Engelhardt and Liebner, 2014). Between E9.5 and E10.5, the first blood vessels from the PNVP start ingressing into the developing SC in a stereotypical growth pattern (Nakao et al., 1988; Himmels et al., 2017). Unlike the SC, where vessels ingress from a single vascular plexus, the neocortex is vascularized by the ingression of vessels from the PNVP and an additional periventricular plexus (PVP) (Vasudevan et al., 2008). There, intrinsic transcriptional factors ( $\mathrm{Nkx} 2.1, \mathrm{Dlx} 1 / 2$, and Pax6) expressed in endothelial cells are crucial for such vessel patterning from the PVP (Vasudevan et al., 2008).

Research from the last decade suggests that during development the nervous and vascular systems actively and vitally crosstalk with each other to build a fully functional organ system (Paredes et al., 2018; Segarra et al., 2019). In the next sections, we will focus on those interactions that occur during SC development in coordination with vascularization. From the current knowledge of developmental studies in SC using different animal models, we also introduce the readers with SC pathologies and the molecular targets discovered during development that could prove beneficial during injuries and diseases.

\section{SPINAL CORD VASCULARIZATION: FROM A SIMPLE PLEXUS TO A COMPLEX NETWORK}

\section{Development of the PNVP}

The early development of the SC vasculature begins at around E8.5 in mouse embryos and around E3 in avian embryos with the formation of the PNVP, a primitive vascular network formed via the process of vasculogenesis (Noden, 1988; Kurz et al., 1996). The assembling of this vascular bed initially requires proliferation, migration, and differentiation of mesoderm-derived angioblasts, a mesenchymal cell type giving rise to the endothelial cell lineage (Noden, 1988; Kurz et al., 1996). The identification of the first key factors contributing for this process, FGF-2 and vascular endothelial growth factor (VEGF), was initially identified by experimentation in quail (Cox and Poole, 2000; Hogan et al., 2004). In avian and mouse embryos, the first primordial vascular structures neighboring the SC and fusing to the PNVP are the dorsal aorta (the major artery) and the cardinal vein (the major vein). Mice and avians present two dorsal aortas, one dorsal aorta in each side of the midline (defined by the notochord) - but fusing into a single artery in the mid-trunk region - and two cardinal veins, similarly one in each side of the midline along the entire body (Hogan and Bautch, 2004; Kurz, 2009). In avians, primitive arterial tracts connect each of the dorsal aortas to the ventral part of the PNVP, laterally to the FP, precisely where the first sprouts from the PNVP ingress into the SC. Afterward, the SC vascular circuit is closed and drained into the cardinal veins, which are connected to the PNVP in the lateral sides of the SC (Kurz, 2009). For the best of our knowledge, a similar vascular circuit in mouse has not been demonstrated yet.

In zebrafish, the PNVP forms, however, a bit different. In zebrafish, the dorsal aorta and the cardinal vein are single vessels located below the notochord and extend throughout the entire anterior-posterior axis. The PNVP surrounding the SC arises by the combination of sprouts from arterial and venous intersegmental vessels (ISVs; which sprout from the previously formed dorsal aorta and posterior cardinal vein) and vertebral arteries (VTAs; Isogai et al., 2001, 2003; Matsuoka et al., 2016, 2017; Wild et al., 2017). Bilateral VTAs are formed along the SC by sprouting from the previously established ISVs. Genetically-ablation studies have shown that this process in zebrafish is regulated by VEGF secreted from radial glia cells located in the SC (Matsuoka et al., 2017). Interestingly, not only radial glia but also SC neurons were also shown to similarly control sprouting of venous ISVs around the developing SC in zebrafish (Wild et al., 2017). SC neurons simultaneously secrete VEGF and SFLT1 and, in case of a shift in the balance toward the former, ectopic sprouting of venous ISVs (not arterial ISVs) arise and surround the SC, suggesting that the VEGF signaling affects specifically venous ECs during this development stage window (Wild et al., 2017).

\section{Vessel Ingression Into the SC}

In avians, single angioblasts are able to invade and migrate into the SC and thus contribute, together with the sprouting from the PNVP, to SC vascularization (Kurz et al., 1996). In mice, in contrast, once the PNVP is formed, vessel ingression into of the SC only occurs from new sprouts arising from the PNVP - via sprouting angiogenesis (Nakao et al., 1988; Himmels et al., 2017).

Blood vessel ingression into the SC occurs in a highly stereotypical way. At around E10.5 in mouse embryos, sprouting blood vessels from the PNVP invade the neural tissue in a very specific pattern: the first sprouts invade the ventral part of the SC in between the floor plate and MN columns and also from the lateral-ventral side in proximity with the MNs (Figure 1). VEGF, demonstrated to be important for PNVP formation, also plays a role in this initial blood vessel ingression. Interestingly, different VEGFA isoforms perturbed SC angiogenesis at different levels. While ectopic expression of VEGFA165 and VEGFA189 in quail embryos resulted in a considerable increase of vessel ingression, only a mild phenotype is observed after ectopic expression of VEGFA121 (James et al., 2009). Although VEGFA plays a role in blood 

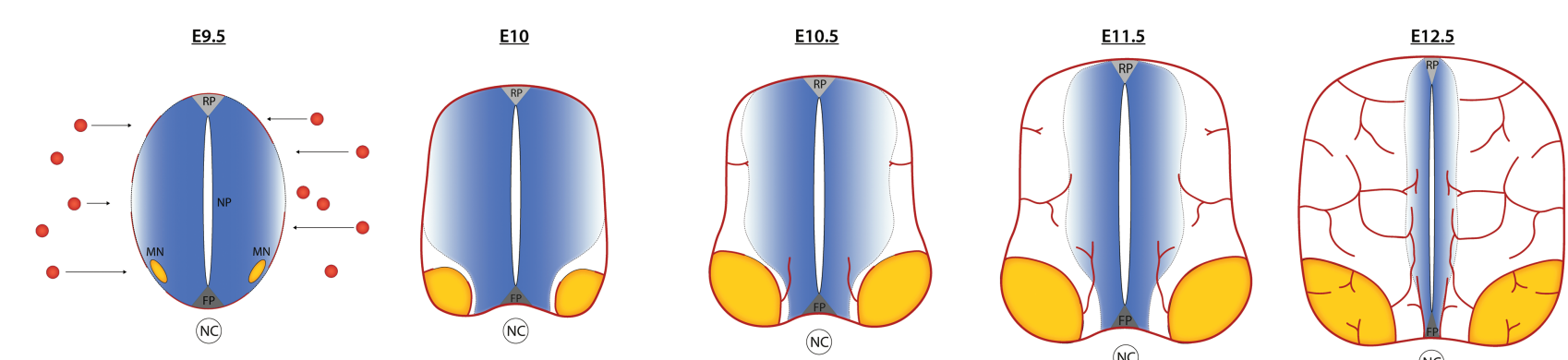

(NC)

Factors involved in spinal cord vascularization

\begin{tabular}{|c|c|c|}
\hline E9.5 - Development of the PNVP & E10 to E10.5 - Vessel ingression into the SC & E11.5 onwards - Stereotypical vessel patterning within the SC \\
\hline $\begin{array}{c}\text { FGF-2 } \\
\text { (Cox and Poole., 2000) } \\
\text { VEGFA } \\
\text { (Hogan et al., 2004; Matsuoka et al., 2017) }\end{array}$ & $\begin{array}{c}\text { WNT7a/b } \\
\text { (Daneman et al., 2009; Stenman et al, 2008) } \\
\text { GPR124 } \\
\begin{array}{c}\text { (Kuhnert et al., 2010; Posokhova et al., 2015; Vanhollebeke et al., 2015; Zhou and Nathans, 2014) } \\
\text { RECK-Norrin } \\
\text { (Cho et al., 2017; Vanhollebeke et al., 2015) } \\
\text { VEGFA } \\
\text { (James et al., 2009) } \\
\text { FFLT1 } \\
\text { (Wild et all., 2017) }\end{array}\end{array}$ & 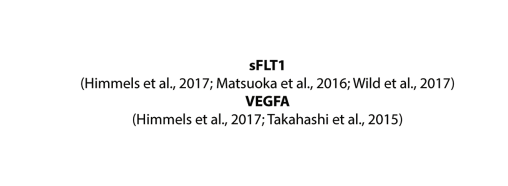 \\
\hline
\end{tabular}

FIGURE 1 | Stereotypical patterning of spinal cord (SC) vascularization. In normal conditions, the avascular SC starts to be vascularized at embryonic day 8.5-9.5 in mice by the formation of the perineural vascular plexus (PNVP). Between E9.5 and E10.5, blood vessels ingressing from the PNVP follow a specific invading pattern: the first vessel sprouts enter between the floor plate (FP) and motor neuron (MN) columns; subsequently they surround the MN columns and continue growing towards dorsal areas, but continuously avoiding the MNs region, the FP and part of the neural progenitors' area (NP) for a particular development time window. At E12.5 MN columns are finally vascularized and a dense network of vessels sustains the continuous growth of the SC. Factors involved in the different steps are indicated in the table.

vessel ingression, its expression is not specifically localized to areas of ingression, instead is broadly expressed in dorsal and ventral areas of the SC. This suggests that VEGFA is necessary but may not be the only factor controlling the location and timing of blood vessel sprouting from the PNVP (James et al., 2009). In fact, recent studies show that sFLT1 expression is also important to control the initial vascular sprouting into the SC in mouse (Himmels et al., 2017) and zebrafish (Wild et al., 2017). SC neurons (MNs in the case of mouse embryos) simultaneously secrete VEGF and sFLT1 to control the regions and timing of vessel ingression from the PNVP (Himmels et al., 2017; Wild et al., 2017).

WNT ligands play a major role in the initial sprouting of vessels into the SC. WNT7a and WNT7b, as other family members of the WNT family, are expressed in specific regions of the SC coincident with the time of ingression of the first vessel sprouts (Parr et al., 1993; Hollyday et al., 1995; Stenman et al., 2008; Daneman et al., 2009). WNT7a/b are expressed by several ventral and dorsal neural progenitors surrounding the ventricle at E10.5 and, while single null mutants for WNT7a or WNT7b do not present any phenotype, WNT7a/b double mutants show defects in their CNS-specific vasculature with the embryos dying around E12.5 (Stenman et al., 2008; Daneman et al., 2009). Remarkably, double mutant embryos were completely devoid of vessels and pericytes in the ventral part of the SC, but presented vessels (however with abnormal morphology) in dorsal regions of the SC. The fact that vessels continue to ingress into dorsal areas after deletion of WNT7a/b might suggested a role in SC vascularization for other WNT ligands expressed by the dorsal domains (Stenman et al., 2008). Consistently, blocking the canonical WNT signaling pathway by the removal of $\beta$-catenin from endothelial cells results in a more severe phenotype with the complete absence of blood vessels in the entire SC (Stenman et al., 2008; Daneman et al., 2009). Interestingly, in those mutants PNVP formation occurs normally, indicating that WNT signaling plays a role in the initial vessel ingression but not in PNVP formation (Stenman et al., 2008). Additionally, WNT7a/b also promote blood-brain barrier (BBB) formation, as lack of WNT7 leads to a reduction of GLUT1, the main glucose transporter in ECs of the CNS (Stenman et al., 2008). Wnt7a/b signal via Frizzled receptors (Zerlin et al., 2008) and RECK (reversion-inducing cysteinerich protein), a GPI-anchored plasma membrane protein, was shown to act as a specific co-receptor for WNT7a/b in ECs (Vanhollebeke et al., 2015; Ulrich et al., 2016; Cho et al., 2017).

GPR124 is expressed by endothelial cells and pericytes and GPR124 null mice present severe hemorrhages as early as E12.5 and embryonic lethality at E15.5 (Kuhnert et al., 2010). Interestingly, GPR124 null mice present the same developmental defects observed in WNT7a/b and $\beta$-catenin null mice, suggesting that both signaling pathways share a common mechanism (Kuhnert et al., 2010). Indeed, independent groups showed that GPR124 acts as a co-activator of WNT7a/b-specific canonical pathway in endothelial cells (Zhou and Nathans, 2014; Posokhova et al., 2015; Vanhollebeke et al., 2015). Further studies have shown that a synergetic action of RECKNorrin-GPR124 receptors in WNT7a/b signaling to promote vascular development, and the absence of either one is enough 
to reduce, but not completely abolish, WNT7a/b signaling (Vanhollebeke et al., 2015; Cho et al., 2017).

\section{Vessel Patterning Within the SC}

As indicated above, the first vessel sprouts invade the ventral part of the SC in between the floor plate and $\mathrm{MN}$ columns and also from the lateral-ventral side in proximity with the MNs (Figure 1). At E11.5, vessels ingressing from both sites migrate toward each other and completely surround the MN columns, whereas at the same time additional sprouts branch from the previous vessels and also extend into dorsal areas of the SC, always along and in close contact with the ventricular zone occupied by the neural progenitors. Initially vessels do not ingress from the most dorsal part of the PNVP (Feeney and Watterson, 1946; James et al., 2009; Himmels et al., 2017). A considerable amount of research has been developed to try to understand this well-defined vascular patterning but still little is known about how the neural cells and growing blood vessels communicate to each other to orchestrate a correct SC vascularization pattern. Interestingly, we and others found that it is in the areas that blood vessels initially avoid (the floor plate, neural progenitors, and MN columns), where the highest amount of VEGF is detected (James et al., 2009; Ruiz de Almodovar et al., 2011; Himmels et al., 2017). This suggested that together with VEGF other factors were involved in blood vessel patterning. In line with this idea, we demonstrated how MNs control their own vascularization during a particular development time window (E10.5-E12.5) by simultaneously secreting VEGF and its decoy receptor sFLT1 in a hypoxiainducible factor 1 (HIF1 $\alpha$ ) and neuropilin-1 (NRP1) dependent manner, respectively (Himmels et al., 2017). The importance of a perfect balance of these molecules is visible when overexpressing of VEGF in neural cells in mouse embryos or when reducing sFLT1 or NRP1 by knocking down their expression using in ovo experiments. Under those different conditions premature blood vessel ingression into MN columns occurs, showing an example of a neural-to-vessel communication mechanism that shapes SC development (Himmels et al., 2017). The importance of the balance VEGF-sFLT1 for a correct SC vascularization has also been described in zebrafish (Matsuoka et al., 2016, 2017; Wild et al., 2017).

As mentioned above blood vessels ingress from the ventral region and migrate toward dorsal areas along a well-defined path: in mice, vessels grow in close contact to the undifferentiated neural progenitors next to the ventricle while in avian vessels extend in between the undifferentiated neural progenitor zone $\left(\right.$ Sox $\left.2^{+}\right)$and the differentiated zone (Sox2- Tuj- $1^{+}$; Takahashi et al., 2015; Himmels et al., 2017). Manipulation of these two areas in avian by locally disturbing neurogenesis originates a change in vascular patterning, suggesting that this particular growth path is controlled by the surrounding neural cells (Takahashi et al., 2015). Further, gain-of-function studies in chick embryos demonstrated that the stereotypical pattern along the neural progenitor area is achieved by the secretion of VEGF from the neural progenitors, attracting growing sprouts, but simultaneously endothelial cell response to VEGF is fine-tuned by sFLT1 secretion by ECs (Takahashi et al., 2015).
Notably, the degree of angiogenesis needs to be controlled as negative effects on the neural compartment can arise when too much VEGF is available. As shown in Himmels et al. (2017), MNs secrete VEGF and sFLT1 simultaneously to ensure that a correct level and timing of angiogenesis takes place. When this balance of factors is disrupted and excessive angiogenesis occurs, MNs are distributed incorrectly and MN axons leave the SC defasciculated (Himmels et al., 2017). When extrapolating this to situations, where axon regrowth is needed, it is possible that simply inducing regeneration of axons and angiogenesis is not enough to promote proper axon regrowth, but that angiogenesis needs to be limited to not disrupt the other process.

With respect to the specific pattern of arteries, veins, and capillaries, it is well-defined that in the adult SC the main arterial supply is achieved by the presence of segmental spinal arteries arising from the vertebral arteries, dorsal intercostal arteries, and lumbar arteries. Blood flows in the SC through these arteries and is further drained into the large and dorsal and posterior spinal veins in the SC (Farrar et al., 2015; Mazensky et al., 2017). Yet, it remains to be further characterized how the pattern of these three different vessel types appears during development.

\section{COMMON MOLECULAR FACTORS IN DEVELOPMENT AND PATHOLOGY}

CNS pathologies are multifaceted and complex pathologies characterized by cell death, axon damage/degeneration, loss of vascular integrity, disruption of the $\mathrm{BBB}$ and blood-spinal cord barrier (BSCB), inflammation, and ECM (extracellular matrix) remodeling (Griffin and Bradke, 2020). The BBB and BSCB are sophisticated barrier systems in which ECs and their tight junctions play a central part in association with astrocyte end-feet, perivascular macrophages, pericytes, and basement lamina (Bartanusz et al., 2011). However, they are heterogenous in concern to their expression of barrier-specific proteins and their functional permeability. Compared to brain, ECs in the SC seem to have decreased expression of adherens junction (AJ) and tight junction (TJs) proteins and show a corresponding increase in permeability to low-molecular-weight tracers (Bartanusz et al., 2011).

It is by now understood that neuronal regeneration events during pathology and embryonic development by NPCs elicit very similar transcriptomic response making them fairly similar processes (Poplawski et al., 2020). Comparably, pathways that regulate vascular development and $\mathrm{BBB}$ properties are also active in pathological conditions. We provide here some examples of these molecules and their signaling pathways that play a significant role during vascularization and $\mathrm{BBB}$ formation in the SC and also during its pathology. Due to space limitations, we took as examples the experimental autoimmune encephalomyelitis (EAE), amyotrophic later sclerosis (ALS), and arteriovenous malformations (AVMs) in the SC.

Among the available experimental models to understand the BSCB pathology, the EAE mouse model, characterized 
by autoimmune attack to oligodendrocytes in the CNS leading to their loss and demyelination of axons, is one of the most commonly used (Tonra et al., 2001; Muller et al., 2005; Schellenberg et al., 2007; Aube et al., 2014). Multiple reports describe changes in endothelial proliferation, vessel morphology, and increased blood vessel density in the SC and brain with EAE (Kirk and Karlik, 2003; Roscoe et al., 2009; Seabrook et al., 2010). In addition, a recent study applying the EAE model in Claudin5-GFP reporter line (with ECs expressing GFP) shows that remodeling of TJs in ECs and paracellular BSCB leakage precedes the EAE disease onset. (Lutz et al., 2017). Changes in VEGF expression and increased levels of VEGF have been described in EAE and in multiple sclerosis (MS) patients (Proescholdt et al., 2002; Su et al., 2006; Shimizu et al., 2012) and may eventually be responsible for the increase in EC proliferation and vessel density as well as for the leaky barrier (Argaw et al., 2009; Luissint et al., 2012). Once the immune system gets activated, it further exacerbates VEGF signaling cascade ending into a feedback loop that would further promote BSCB leakage and inflammation (Argaw et al., 2006, 2012). Based on this increase in VEGF expression, one of the strategies to balance the VEGF availability, learned from developmental studies, would be to promote the expression of sFLT1 that could titrate the excess of VEGF from the system (Himmels et al., 2017; Wild et al., 2017).

As mentioned above, WNT ligands are known for their specific role in CNS angiogenesis and BBB formation (Stenman et al., 2008; Daneman et al., 2009). Consistent with its role as a co-receptor for WNT ligands, GPR124 also participates in BBB formation (Kuhnert et al., 2010; Anderson et al., 2011; Cullen et al., 2011). The WNT/ $\beta$-catenin signaling pathway is important for adult BBB maintenance as shown in multiple reports (Tran et al., 2016; Chang et al., 2017; Griveau et al., 2018) and is activated in CNS endothelium also in EAE and human MS during the course of disease progression (Lengfeld et al., 2017; Niu et al., 2019). The re-activation of this pathway in pathological conditions may suggest a rapid endothelial response toward restoring the barrier properties of the damaged vessels. Consistent with this hypothesis, in vivo inhibition of WNT signaling in ECs exacerbated EAE pathology with increased mortality, greater infiltration of $\mathrm{CD} 4+\mathrm{T}$ cells into the CNS and more drastic myelin loss (Lengfeld et al., 2017). Interestingly, in postnatal or adult mice conditional deletion of endothelial GPR124 resulted in no defects in CNS angiogenesis, BBB development or maintenance; making GPR124 dispensable for vascular homeostasis in adult CNS (Chang et al., 2017). However, deficiency of GPR124 in a pathological mouse model of ischemic stroke or glioblastoma leads to extensive BBB leakage and hemorrhage, microvascular damage accompanied by pericyte, ECM, and TJ deficits. Thus, similar as the re-activation of the WNT signaling pathway, the GPR124-WNT signaling axis is important in maintaining vascular homeostasis during injury in adult. Considering that BSCB leakage is a primary feature of several diseases, one approach to target leakage, suggested by those studies, and by the role of the WNT/ $\beta$ catenin signaling in BBB formation during development, could be to promote the activation of this pathway in ECs in pathological conditions (Liu et al., 2014; Jia et al., 2019). This was already shown in the above mentioned GPR124 deletion mouse pathology-model, where EC-specific constitutive activation of WNT signaling via activated $\beta$-catenin restored the vascular defects (Chang et al., 2017). In line with this idea, it was recently also shown that the activation of $\beta$-catenin in ECs from circumventricular organs of the CNS, which under physiological conditions lack $\mathrm{BBB}$ properties and are permeable, results in a tightened $\mathrm{BBB}$ in those regions and augmented neuronal activity (Benz et al., 2019).

Amyotrophic lateral sclerosis (ALS) is another SC and brain related disease, where the progression of $\mathrm{MN}$ degeneration leads to muscle atrophy, paralysis, and death. In ALS, BSCB impairment is also shown (Taylor et al., 2016). The disruption of endothelial TJ proteins like ZO1, occludin, and claudin5 seems to be the primary cause of microhemorrhages, reduced microcirculation, prior to the $\mathrm{MN}$ degeneration and the inflammatory response (Zhong et al., 2008). There is increasing evidence suggesting that $\mathrm{MN}$ degeneration is not only due to intrinsic defects, but also that the surrounding cell types like microglia, astrocytes, oligodendrocytes, and ECs may also be involved. A variety of growth and neurotrophic factors are also reported to mediate the ALS pathology (Bruijn et al., 2004). Of note, VEGF and WNTs are well studied. Mice with reduced VEGF levels (Vegf/s mice) present reduced neural vascular perfusion and progressive MN degeneration, mimicking the human ALS (Oosthuyse et al., 2001). Multiple studies have shown the importance of VEGF in decelerating the disease outcome and providing a protective effect for MNs by both maintaining proper vessel perfusion and by acting directly on MNs as a survival factor (Lange et al., 2016). The underlying pathophysiological mechanisms leading to the MN degeneration and fatal outcome observed in ALS also seems to be linked to WNTs (Chen et al., 2012; Yu et al., 2013; Tury et al., 2014; Gonzalez-Fernandez et al., 2016, 2020; Bhinge et al., 2017). SOD1 ${ }^{\mathrm{G} 93 \mathrm{~A}}$ ALS mice, as well as ALS patients, present a dysregulation in WNT signaling with upregulation or downregulation of certain ligands, receptors, and coreceptors depending on the distinct cell of the CNS analyzed (Gonzalez-Fernandez et al., 2020). As WNT ligands can act and exert different functions in different cellular context, close examination of their effect in astrocytes, neurons, and blood vessels in ALS conditions is required to understand their overall outcome. In this regard, upregulation of canonical WNT signaling seems to promote glial proliferation, that is, eventually neuroprotective during ALS (Chen et al., 2012; Li et al., 2013; Yu et al., 2013). In a similar manner, extrapolating from their role in blood vessel and BBB formation in the CNS, upregulated WNT ligands could be a defense mechanism to also promote a tighter $\mathrm{BBB}$. However, their role in conjunction with the CNS vessels in this pathology needs further investigation.

Arteriovenous malformations (AVMs) are characterized by abnormal tangles of blood vessels connecting arteries and veins, where blood flow is thus shunted from arteries to veins without 
passing through a capillary network (Mouchtouris et al., 2015). While AVMs can develop anywhere in our body, they occur most often in the CNS (being more common in the brain than in the SC). Spinal AVMs are mainly represented during the adulthood, however, they may also appear as a juvenile form, which can be intramedullary, extramedullary, and extraspinal (Ferch et al., 2001). Most of the molecular studies on CNS AVMs have been in the brain and indicate that similar mechanisms involved in CNS vascularization are de-regulated in AVMs. Although not described in the SC AVMs, here, we will describe those main findings.

Multiple studies suggest active angiogenesis as a feature of AVMs. Similarly, inflammation is a major contributor toward the pathogenesis and active angiogenesis of AVMs. The inflammatory response triggered by hemodynamic factors and/or genetic predisposition in the formation and rupture of AVMs involves different cytokines (IL-6, IL-1 $\beta, \mathrm{TNF} \alpha$, and IL-8), neutrophils, and macrophages (Mouchtouris et al., 2015) that eventually upregulate the expression of VEGF. IL-6, IL-1 $\beta$, and TNF $\alpha$ also induce NF-kB expression, which further exacerbates VEGF levels (Angelo and Kurzrock, 2007). Moreover, it is possible that this AVM niche itself may contribute to the stimulation of pathological angiogenesis, where the occurrence of focal ischemia leads to a hypoxic environment that in turn leads to angiogenesis by the expression of VEGF and VEGFR1 (Rothbart et al., 1996; Koizumi et al., 2002; Jabbour et al., 2009; Mouchtouris et al., 2015; Gamboa et al., 2017). Worth to mention, the NF-kB-VEGF cascade in AVMs is also stimulated under hypoxia by HIF-1 (Ng et al., 2005). Consistently, VEGF has been reported in patients suffering from recurrent cerebral AVMs (Rothbart et al., 1996) and VEGF receptors (VEGFR1 and VEGFR2), matrix metalloproteinases-MMP9 and ECM proteins like Collagen IV (Rothbart et al., 1996; Pyo et al., 2000; Hashimoto et al., 2003; Gamboa et al., 2017) are further thought to play a primary role in the pathogenesis of AVMs. As Notch signaling plays a critical role in the determining the arterio-venous cell fate during vascular development, it is thus crucial in AVMs also (Murphy et al., 2008; ZhuGe et al., 2009; Tetzlaff and Fischer, 2018). Manipulations in the notch signaling pathway leads to development of hallmark features of AVMs in the brain (Murphy et al., 2008; Nielsen et al., 2014). Recently a few studies have also shed light on the role of Wnt signaling in AVMs. $\beta$-catenin gain-of-function in ECs can cause arterial defects, including the loss of venous marker expression, arterialization of veins, and formation of AVMs (Corada et al., 2010). These ECs show a strong increase in

\section{REFERENCES}

Anderson, K. D., Pan, L., Yang, X. M., Hughes, V. C., Walls, J. R., Dominguez, M. G., et al. (2011). Angiogenic sprouting into neural tissue requires Gpr124, an orphan G protein-coupled receptor. Proc. Natl. Acad. Sci. U. S. A. 108, 2807-2812. doi: 10.1073/pnas.1019761108

Angelo, L. S., and Kurzrock, R. (2007). Vascular endothelial growth factor and its relationship to inflammatory mediators. Clin. Cancer Res. 13, 2825-2830. doi: 10.1158/1078-0432.CCR-06-2416
Dll4/Notch signaling along with reduced sprouting activity, indicating a requirement for fine-tuning Wnt and Notch signaling pathways in the pathogenesis of AVMs. Additionally, a very recent study on whole-exome RNA sequencing in human samples of brain AVMs also showed an activation of canonical Wnt signaling via the activity of increased FZD10 and MYOC (Huo et al., 2019). All in all, the current understanding in AVM pathology, diagnoses, and treatment is increasing, but the molecular players and its regulation require further investigation. In addition, whether the same molecular mechanisms that lead to AVMs in the brain are also active in spinal AVMs is also something that remains to be investigated.

These examples show that VEGF and WNT signaling, that are important for blood vessel growth and $\mathrm{BBB}$ formation, during development are also involved in distinct pathologies.

\section{CONCLUDING REMARKS}

Research in the past recent years has provided further mechanistic insight into neuro-vascular interactions and into the importance of those interactions for proper development and function of the CNS. Here, studies in the SC have been fundamental. To advance in our understanding of SC development and function further studies focused on delineating angiogenic cues and potential bidirectional signaling pathways are needed. Spinal cord injuries are debilitating and fatal in many cases. Understanding SC development from a neuralvascular interaction perspective, and the potential reactivation or inhibition of those molecular signaling pathways in pathological conditions will be important for further developing therapeutic strategies.

\section{AUTHOR CONTRIBUTIONS}

JRV, BS, and CRA wrote the manuscript. All authors contributed to the article and approved the submitted version.

\section{FUNDING}

This work was supported by the EU: ERC-CoG grant (ref: 864875) and the DFG: DFG grants from SFB1366 (project number 394046768-SFB 1366), and from SFB 1158. 
of the hypoxia-angiogenesis program. J. Immunol. 177, 5574-5584. doi: 10.4049/jimmunol.177.8.5574

Aube, B., Levesque, S. A., Pare, A., Chamma, E., Kebir, H., Gorina, R., et al. (2014). Neutrophils mediate blood-spinal cord barrier disruption in demyelinating neuroinflammatory diseases. J. Immunol. 193, 2438-2454. doi: 10.4049/jimmunol.1400401

Bartanusz, V., Jezova, D., Alajajian, B., and Digicaylioglu, M. (2011). The bloodspinal cord barrier: morphology and clinical implications. Ann. Neurol. 70, 194-206. doi: 10.1002/ana.22421

Benz, F., Wichitnaowarat, V., Lehmann, M., Germano, R. F., Mihova, D., Macas, J., et al. (2019). Low wnt/beta-catenin signaling determines leaky vessels in the subfornical organ and affects water homeostasis in mice. elife 8:e43818. doi: 10.7554/eLife.43818

Bhinge, A., Namboori, S. C., Zhang, X., VanDongen, A. M. J., and Stanton, L. W. (2017). Genetic correction of SOD1 mutant iPSCs reveals ERK and JNK activated AP1 as a driver of neurodegeneration in amyotrophic lateral sclerosis. Stem Cell Reports. 8, 856-869. doi: 10.1016/j.stemcr.2017.02.019

Bruijn, L. I., Miller, T. M., and Cleveland, D. W. (2004). Unraveling the mechanisms involved in motor neuron degeneration in ALS. Annu. Rev. Neurosci. 27, 723-749. doi: 10.1146/annurev.neuro.27.070203.144244

Chang, J., Mancuso, M. R., Maier, C., Liang, X., Yuki, K., Yang, L., et al. (2017). Gpr124 is essential for blood-brain barrier integrity in central nervous system disease. Nat. Med. 23, 450-460. doi: 10.1038/nm.4309

Chen, Y., Guan, Y., Liu, H., Wu, X., Yu, L., Wang, S., et al. (2012). Activation of the Wnt/beta-catenin signaling pathway is associated with glial proliferation in the adult spinal cord of ALS transgenic mice. Biochem. Biophys. Res. Commun. 420, 397-403. doi: 10.1016/j.bbrc.2012.03.006

Cho, C., Smallwood, P. M., and Nathans, J. (2017). Reck and Gpr124 are essential receptor cofactors for Wnt7a/Wnt7b-specific signaling in mammalian CNS angiogenesis and blood-brain barrier regulation. Neuron 95, 1056-1073. doi: $10.1016 /$ j.neuron.2017.07.031

Corada, M., Nyqvist, D., Orsenigo, F., Caprini, A., Giampietro, C., Taketo, M. M., et al. (2010). The Wnt/beta-catenin pathway modulates vascular remodeling and specification by upregulating Dll4/notch signaling. Dev. Cell 18, 938-949. doi: $10.1016 /$ j.devcel.2010.05.006

Cox, C. M., and Poole, T. J. (2000). Angioblast differentiation is influenced by the local environment: FGF-2 induces angioblasts and patterns vessel formation in the quail embryo. Dev. Dyn. 218, 371-382. doi: 10.1002/(SIC I) 1097-0177(200006)218:2<371::AID-DVDY10>3.0.CO;2-Z

Cullen, M., Elzarrad, M. K., Seaman, S., Zudaire, E., Stevens, J., Yang, M. Y., et al. (2011). GPR124, an orphan G protein-coupled receptor, is required for CNS-specific vascularization and establishment of the blood-brain barrier. Proc. Natl. Acad. Sci. U. S. A. 108, 5759-5764. doi: 10.1073/pnas.1017192108

Daneman, R., Agalliu, D., Zhou, L., Kuhnert, F., Kuo, C. J., and Barres, B. A. (2009). Wnt/beta-catenin signaling is required for CNS, but not non-CNS, angiogenesis. Proc. Natl. Acad. Sci. U. S. A. 106, 641-646. doi: 10.1073/ pnas. 0805165106

Dessaud, E., McMahon, A. P., and Briscoe, J. (2008). Pattern formation in the vertebrate neural tube: a sonic hedgehog morphogen-regulated transcriptional network. Development 135, 2489-2503. doi: 10.1242/dev.009324

Dessaud, E., Yang, L. L., Hill, K., Cox, B., Ulloa, F., Ribeiro, A., et al. (2007). Interpretation of the sonic hedgehog morphogen gradient by a temporal adaptation mechanism. Nature 450, 717-720. doi: 10.1038/nature06347

Diez Del Corral, R., and Morales, A. V. (2017). The multiple roles of FGF signaling in the developing spinal cord. Front. Cell Dev. Biol. 5:58. doi: 10.3389/fcell.2017.00058

Diez del Corral, R., Olivera-Martinez, I., Goriely, A., Gale, E., Maden, M., and Storey, K. (2003). Opposing FGF and retinoid pathways control ventral neural pattern, neuronal differentiation, and segmentation during body axis extension. Neuron 40, 65-79. doi: 10.1016/s0896-6273(03)00565-8

Duester, G. (2008). Retinoic acid synthesis and signaling during early organogenesis. Cell 134, 921-931. doi: 10.1016/j.cell.2008.09.002

Engelhardt, B., and Liebner, S. (2014). Novel insights into the development and maintenance of the blood-brain barrier. Cell Tissue Res. 355, 687-699. doi: 10.1007/s00441-014-1811-2

Farrar, M. J., Rubin, J. D., Diago, D. M., and Schaffer, C. B. (2015). Characterization of blood flow in the mouse dorsal spinal venous system before and after dorsal spinal vein occlusion. J. Cereb. Blood Flow Metab. 35, 667-675. doi: $10.1038 /$ jcbfm. 2014.244
Feeney, J. F. Jr., and Watterson, R. L. (1946). The development of the vascular pattern within the walls of the central nervous system of the chick embryo. J. Morphol. 78, 231-303. doi: 10.1002/jmor.1050780205

Ferch, R. D., Morgan, M. K., and Sears, W. R. (2001). Spinal arteriovenous malformations: a review with case illustrations. J. Clin. Neurosci. 8, 299-304. doi: 10.1054/jocn.2000.0914

Gamboa, N. T., Taussky, P., Park, M. S., Couldwell, W. T., Mahan, M. A., and Kalani, M. Y. S. (2017). Neurovascular patterning cues and implications for central and peripheral neurological disease. Surg. Neurol. Int. 8:208. doi: 10.4103/sni.sni_475_16

Gonzalez-Fernandez, C., Gonzalez, P., and Rodriguez, F. J. (2020). New insights into Wnt signaling alterations in amyotrophic lateral sclerosis: a potential therapeutic target? Neural Regen. Res. 15, 1580-1589. doi: 10.4103/16735374.276320

Gonzalez-Fernandez, C., Mancuso, R., Del Valle, J., Navarro, X., and Rodriguez, F. J. (2016). Wnt signaling alteration in the spinal cord of amyotrophic lateral sclerosis transgenic mice: special focus on Frizzled-5 cellular expression pattern. PLoS One 11:e0155867. doi: 10.1371/journal.pone.0155867

Griffin, J. M., and Bradke, F. (2020). Therapeutic repair for spinal cord injury: combinatory approaches to address a multifaceted problem. EMBO Mol. Med. 12:e11505. doi: 10.15252/emmm.201911505

Griveau, A., Seano, G., Shelton, S. J., Kupp, R., Jahangiri, A., Obernier, K., et al. (2018). A glial signature and Wnt7 signaling regulate glioma-vascular interactions and tumor microenvironment. Cancer Cell 33, 874-889 e7. doi: 10.1016/j.ccell.2018.03.020

Hashimoto, T., Wen, G., Lawton, M. T., Boudreau, N. J., Bollen, A. W., Yang, G. Y., et al. (2003). Abnormal expression of matrix metalloproteinases and tissue inhibitors of metalloproteinases in brain arteriovenous malformations. Stroke 34, 925-931. doi: 10.1161/01.STR.0000061888.71524.DF

Himmels, P., Paredes, I., Adler, H., Karakatsani, A., Luck, R., Marti, H. H., et al. (2017). Motor neurons control blood vessel patterning in the developing spinal cord. Nat. Commun. 8:14583. doi: 10.1038/ncomms14583

Hogan, K. A., Ambler, C. A., Chapman, D. L., and Bautch, V. L. (2004). The neural tube patterns vessels developmentally using the VEGF signaling pathway. Development 131, 1503-1513. doi: 10.1242/dev.01039

Hogan, K. A., and Bautch, V. L. (2004). Blood vessel patterning at the embryonic midline. Curr. Top. Dev. Biol. 62, 55-85. doi: 10.1016/S0070-2153(04)62003-5

Hollyday, M., McMahon, J. A., and McMahon, A. P. (1995). Wnt expression patterns in chick embryo nervous system. Mech. Dev. 52, 9-25. doi: 10.1016/0925-4773(95)00385-E

Huo, R., Fu, W., Li, H., Jiao, Y., Yan, Z., Wang, L., et al. (2019). RNA sequencing reveals the activation of Wnt signaling in low flow rate brain arteriovenous malformations. J. Am. Heart Assoc. 8:e012746. doi: 10.1161/ JAHA.119.012746

Isogai, S., Horiguchi, M., and Weinstein, B. M. (2001). The vascular anatomy of the developing zebrafish: an atlas of embryonic and early larval development. Dev. Biol. 230, 278-301. doi: 10.1006/dbio.2000.9995

Isogai, S., Lawson, N. D., Torrealday, S., Horiguchi, M., and Weinstein, B. M. (2003). Angiogenic network formation in the developing vertebrate trunk. Development 130, 5281-5290. doi: 10.1242/dev.00733

Jabbour, M. N., Elder, J. B., Samuelson, C. G., Khashabi, S., Hofman, F. M., Giannotta, S. L., et al. (2009). Aberrant angiogenic characteristics of human brain arteriovenous malformation endothelial cells. Neurosurgery 64, 139-146. doi: 10.1227/01.NEU.0000334417.56742.24

James, J. M., Gewolb, C., and Bautch, V. L. (2009). Neurovascular development uses VEGF-A signaling to regulate blood vessel ingression into the neural tube. Development 136, 833-841. doi: 10.1242/dev.028845

Jessell, T. M. (2000). Neuronal specification in the spinal cord: inductive signals and transcriptional codes. Nat. Rev. Genet. 1, 20-29. doi: 10.1038/35049541

Jia, L., Pina-Crespo, J., and Li, Y. (2019). Restoring Wnt/beta-catenin signaling is a promising therapeutic strategy for Alzheimer's disease. Mol. Brain 12: 104. doi: 10.1186/s13041-019-0525-5

Kirk, S. L., and Karlik, S. J. (2003). VEGF and vascular changes in chronic neuroinflammation. J. Autoimmun. 21, 353-363. doi: 10.1016/S0896-8411 (03)00139-2

Koizumi, T., Shiraishi, T., Hagihara, N., Tabuchi, K., Hayashi, T., and Kawano, T. (2002). Expression of vascular endothelial growth factors and their receptors in and around intracranial arteriovenous malformations. Neurosurgery 50, 117-124. doi: 10.1097/00006123-200201000-00020 
Kuhnert, F., Mancuso, M. R., Shamloo, A., Wang, H. T., Choksi, V., Florek, M., et al. (2010). Essential regulation of CNS angiogenesis by the orphan G protein-coupled receptor GPR124. Science 330, 985-989. doi: 10.1126/ science. 1196554

Kurz, H. (2009). Cell lineages and early patterns of embryonic CNS vascularization. Cell Adhes. Migr. 3, 205-210. doi: 10.4161/cam.3.2.7855

Kurz, H., Gartner, T., Eggli, P. S., and Christ, B. (1996). First blood vessels in the avian neural tube are formed by a combination of dorsal angioblast immigration and ventral sprouting of endothelial cells. Dev. Biol. 173, 133-147. doi: 10.1006/dbio.1996.0012

Lange, C., Storkebaum, E., de Almodovar, C. R., Dewerchin, M., and Carmeliet, P. (2016). Vascular endothelial growth factor: a neurovascular target in neurological diseases. Nat. Rev. Neurol. 12, 439-454. doi: 10.1038/nrneurol. 2016.88

Lengfeld, J. E., Lutz, S. E., Smith, J. R., Diaconu, C., Scott, C., Kofman, S. B., et al. (2017). Endothelial Wnt/beta-catenin signaling reduces immune cell infiltration in multiple sclerosis. Proc. Natl. Acad. Sci. U. S. A. 114, E1168-E1177. doi: 10.1073/pnas.1609905114

Li, X., Guan, Y., Chen, Y., Zhang, C., Shi, C., Zhou, F., et al. (2013). Expression of Wnt5a and its receptor Fzd2 is changed in the spinal cord of adult amyotrophic lateral sclerosis transgenic mice. Int. J. Clin. Exp. Pathol. 6, 1245-1260.

Liu, L., Wan, W., Xia, S., Kalionis, B., and Li, Y. (2014). Dysfunctional Wnt/ beta-catenin signaling contributes to blood-brain barrier breakdown in Alzheimer's disease. Neurochem. Int. 75, 19-25. doi: 10.1016/j.neuint.2014.05.004

Luissint, A. C., Artus, C., Glacial, F., Ganeshamoorthy, K., and Couraud, P. O. (2012). Tight junctions at the blood brain barrier: physiological architecture and disease-associated dysregulation. Fluids Barriers CNS. 9:23. doi: 10.1186/ 2045-8118-9-23

Lumsden, A., and Krumlauf, R. (1996). Patterning the vertebrate neuraxis. Science 274, 1109-1115. doi: 10.1126/science.274.5290.1109

Lutz, S. E., Smith, J. R., Kim, D. H., Olson, C. V. L., Ellefsen, K., Bates, J. M., et al. (2017). Caveolin1 is required for Th1 cell infiltration, but not tight junction remodeling, at the blood-brain barrier in autoimmune neuroinflammation. Cell Rep. 21, 2104-2117. doi: 10.1016/j.celrep.2017.10.094

Matsuoka, R. L., Marass, M., Avdesh, A., Helker, C. S., Maischein, H. M., Grosse, A. S., et al. (2016). Radial glia regulate vascular patterning around the developing spinal cord. elife 5:e20253. doi: 10.7554/eLife.20253

Matsuoka, R. L., Rossi, A., Stone, O. A., and Stainier, D. Y. R. (2017). CNSresident progenitors direct the vascularization of neighboring tissues. Proc. Natl. Acad. Sci. U. S. A. 114, 10137-10142. doi: 10.1073/pnas.1619300114

Mazensky, D., Flesarova, S., and Sulla, I. (2017). Arterial blood supply to the spinal cord in animal models of spinal cord injury. A review. Anat. Rec. 300, 2091-2106. doi: 10.1002/ar.23694

Mouchtouris, N., Jabbour, P. M., Starke, R. M., Hasan, D. M., Zanaty, M., Theofanis, T., et al. (2015). Biology of cerebral arteriovenous malformations with a focus on inflammation. J. Cereb. Blood Flow Metab. 35, 167-175. doi: $10.1038 /$ jcbfm.2014.179

Muller, D. M., Pender, M. P., and Greer, J. M. (2005). Blood-brain barrier disruption and lesion localisation in experimental autoimmune encephalomyelitis with predominant cerebellar and brainstem involvement. J. Neuroimmunol. 160, 162-169. doi: 10.1016/j.jneuroim.2004.11.011

Murphy, P. A., Lam, M. T., Wu, X., Kim, T. N., Vartanian, S. M., Bollen, A. W., et al. (2008). Endothelial Notch4 signaling induces hallmarks of brain arteriovenous malformations in mice. Proc. Natl. Acad. Sci. U. S. A. 105, 10901-10906. doi: 10.1073/pnas.0802743105

Nakao, T., Ishizawa, A., and Ogawa, R. (1988). Observations of vascularization in the spinal cord of mouse embryos, with special reference to development of boundary membranes and perivascular spaces. Anat. Rec. 221, 663-677. doi: 10.1002/ar.1092210212

Ng, I., Tan, W. L., Ng, P. Y., and Lim, J. (2005). Hypoxia inducible factor-1alpha and expression of vascular endothelial growth factor and its receptors in cerebral arteriovenous malformations. J. Clin. Neurosci. 12, 794-799. doi: 10.1016/j.jocn.2005.02.005

Nielsen, C. M., Cuervo, H., Ding, V. W., Kong, Y., Huang, E. J., and Wang, R. A. (2014). Deletion of Rbpj from postnatal endothelium leads to abnormal arteriovenous shunting in mice. Development 141, 3782-3792. doi: 10.1242/ dev.108951

Niu, J., Tsai, H. H., Hoi, K. K., Huang, N., Yu, G., Kim, K., et al. (2019). Aberrant oligodendroglial-vascular interactions disrupt the blood-brain barrier, triggering CNS inflammation. Nat. Neurosci. 22, 709-718. doi: 10.1038/ s41593-019-0369-4

Noden, D. M. (1988). Interactions and fates of avian craniofacial mesenchyme. Development 103, 121-140.

Oosthuyse, B., Moons, L., Storkebaum, E., Beck, H., Nuyens, D., Brusselmans, K., et al. (2001). Deletion of the hypoxia-response element in the vascular endothelial growth factor promoter causes motor neuron degeneration. Nat. Genet. 28, 131-138. doi: 10.1038/88842

Paredes, I., Himmels, P., and Ruiz de Almodovar, C. (2018). Neurovascular communication during CNS development. Dev. Cell 45, 10-32. doi: 10.1016/j. devcel.2018.01.023

Parr, B. A., Shea, M. J., Vassileva, G., and McMahon, A. P. (1993). Mouse Wnt genes exhibit discrete domains of expression in the early embryonic CNS and limb buds. Development 119, 247-261.

Poplawski, G. H. D., Kawaguchi, R., Van Niekerk, E., Lu, P., Mehta, N., Canete, P., et al. (2020). Injured adult neurons regress to an embryonic transcriptional growth state. Nature 581, 77-82. doi: 10.1038/s41586-020-2200-5

Posokhova, E., Shukla, A., Seaman, S., Volate, S., Hilton, M. B., Wu, B., et al. (2015). GPR124 functions as a WNT7-specific coactivator of canonical betacatenin signaling. Cell Rep. 10, 123-130. doi: 10.1016/j.celrep.2014.12.020

Proescholdt, M. A., Jacobson, S., Tresser, N., Oldfield, E. H., and Merrill, M. J. (2002). Vascular endothelial growth factor is expressed in multiple sclerosis plaques and can induce inflammatory lesions in experimental allergic encephalomyelitis rats. J. Neuropathol. Exp. Neurol. 61, 914-925. doi: 10.1093/ jnen/61.10.914

Purves, D. (2008). Neuroscience. Sinauer, Sunderland, Mass. xvii, 857, G-816, IC-857, I-829 p.

Pyo, R., Lee, J. K., Shipley, J. M., Curci, J. A., Mao, D., Ziporin, S. J., et al. (2000). Targeted gene disruption of matrix metalloproteinase-9 (gelatinase B) suppresses development of experimental abdominal aortic aneurysms. J. Clin. Invest. 105, 1641-1649. doi: 10.1172/JCI8931

Roscoe, W. A., Welsh, M. E., Carter, D. E., and Karlik, S. J. (2009). VEGF and angiogenesis in acute and chronic MOG((35-55)) peptide induced EAE. J. Neuroimmunol. 209, 6-15. doi: 10.1016/j.jneuroim.2009.01.009

Rothbart, D., Awad, I. A., Lee, J., Kim, J., Harbaugh, R., and Criscuolo, G. R. (1996). Expression of angiogenic factors and structural proteins in central nervous system vascular malformations. Neurosurgery 38, 915-924. doi: 10.1097/00006123-199605000-00011

Ruiz de Almodovar, C., Fabre, P. J., Knevels, E., Coulon, C., Segura, I., Haddick, P. C., et al. (2011). VEGF mediates commissural axon chemoattraction through its receptor Flk1. Neuron 70, 966-978. doi: 10.1016/j.neuron.2011.04.014

Sagner, A., and Briscoe, J. (2019). Establishing neuronal diversity in the spinal cord: a time and a place. Development 146:dev182154. doi: 10.1242/dev.182154

Schellenberg, A. E., Buist, R., Yong, V. W., Del Bigio, M. R., and Peeling, J. (2007). Magnetic resonance imaging of blood-spinal cord barrier disruption in mice with experimental autoimmune encephalomyelitis. Magn. Reson. Med. 58, 298-305. doi: 10.1002/mrm.21289

Seabrook, T. J., Littlewood-Evans, A., Brinkmann, V., Pollinger, B., Schnell, C., and Hiestand, P. C. (2010). Angiogenesis is present in experimental autoimmune encephalomyelitis and pro-angiogenic factors are increased in multiple sclerosis lesions. J. Neuroinflammation 7:95. doi: 10.1186/1742-2094-7-95

Segarra, M., Aburto, M. R., Hefendehl, J., and Acker-Palmer, A. (2019). Neurovascular interactions in the nervous system. Annu. Rev. Cell Dev. Biol. 35, 615-635. doi: 10.1146/annurev-cellbio-100818-125142

Shimizu, F., Sano, Y., Takahashi, T., Haruki, H., Saito, K., Koga, M., et al. (2012). Sera from neuromyelitis optica patients disrupt the blood-brain barrier. J. Neurol. Neurosurg. Psychiatry 83, 288-297. doi: 10.1136/ jnnp-2011-300434

Stenman, J. M., Rajagopal, J., Carroll, T. J., Ishibashi, M., McMahon, J., and McMahon, A. P. (2008). Canonical Wnt signaling regulates organ-specific assembly and differentiation of CNS vasculature. Science 322, 1247-1250. doi: 10.1126/science.1164594

Su, J. J., Osoegawa, M., Matsuoka, T., Minohara, M., Tanaka, M., Ishizu, T., et al. (2006). Upregulation of vascular growth factors in multiple sclerosis: correlation with MRI findings. J. Neurol. Sci. 243, 21-30. doi: 10.1016/j.jns.2005.11.006

Takahashi, T., Takase, Y., Yoshino, T., Saito, D., Tadokoro, R., and Takahashi, Y. (2015). Angiogenesis in the developing spinal cord: blood vessel exclusion from neural progenitor region is mediated by VEGF and its antagonists. PLoS One 10:e0116119. doi: 10.1371/journal.pone.0116119 
Taylor, J. P., Brown, R. H. Jr., and Cleveland, D. W. (2016). Decoding ALS: from genes to mechanism. Nature 539, 197-206. doi: 10.1038/nature20413

Tetzlaff, F., and Fischer, A. (2018). Control of blood vessel formation by notch signaling. Adv. Exp. Med. Biol. 1066, 319-338. doi: 10.1007/ 978-3-319-89512-3_16

Tonra, J. R., Reiseter, B. S., Kolbeck, R., Nagashima, K., Robertson, R., Keyt, B., et al. (2001). Comparison of the timing of acute blood-brain barrier breakdown to rabbit immunoglobulin $\mathrm{G}$ in the cerebellum and spinal cord of mice with experimental autoimmune encephalomyelitis. J. Comp. Neurol. 430, 131-144. doi: 10.1002/1096-9861(20010129)430:1<131::AID-CNE1019>3.0.CO;2-K

Tran, K. A., Zhang, X., Predescu, D., Huang, X., Machado, R. F., Gothert, J. R., et al. (2016). Endothelial beta-catenin signaling is required for maintaining adult blood-brain barrier integrity and central nervous system homeostasis. Circulation 133, 177-186. doi: 10.1161/CIRCULATIONAHA.115.015982

Tury, A., Tolentino, K., and Zou, Y. (2014). Altered expression of atypical PKC and Ryk in the spinal cord of a mouse model of amyotrophic lateral sclerosis. Dev. Neurobiol. 74, 839-850. doi: 10.1002/dneu.22137

Ulrich, F., Carretero-Ortega, J., Menendez, J., Narvaez, C., Sun, B., Lancaster, E., et al. (2016). Reck enables cerebrovascular development by promoting canonical Wnt signaling. Development 143, 147-159. doi: 10.1242/dev.123059

Vanhollebeke, B., Stone, O. A., Bostaille, N., Cho, C., Zhou, Y., Maquet, E., et al. (2015). Tip cell-specific requirement for an atypical Gpr124- and reck-dependent Wnt/beta-catenin pathway during brain angiogenesis. elife 4:e06489. doi: 10.7554/eLife.06489

Vasudevan, A., Long, J. E., Crandall, J. E., Rubenstein, J. L., and Bhide, P. G. (2008). Compartment-specific transcription factors orchestrate angiogenesis gradients in the embryonic brain. Nat. Neurosci. 11, 429-439. doi: 10.1038/ nn2074

Wild, R., Klems, A., Takamiya, M., Hayashi, Y., Strahle, U., Ando, K., et al. (2017). Neuronal sFlt1 and Vegfaa determine venous sprouting and spinal cord vascularization. Nat. Commun. 8:13991. doi: 10.1038/ncomms13991
Wilson, L., and Maden, M. (2005). The mechanisms of dorsoventral patterning in the vertebrate neural tube. Dev. Biol. 282, 1-13. doi: 10.1016/j. ydbio.2005.02.027

Yu, L., Guan, Y., Wu, X., Chen, Y., Liu, Z., Du, H., et al. (2013). Wnt signaling is altered by spinal cord neuronal dysfunction in amyotrophic lateral sclerosis transgenic mice. Neurochem. Res. 38, 1904-1913. doi: 10.1007/ s11064-013-1096-y

Zerlin, M., Julius, M. A., and Kitajewski, J. (2008). Wnt/frizzled signaling in angiogenesis. Angiogenesis 11, 63-69. doi: 10.1007/s10456-008-9095-3

Zhong, Z., Deane, R., Ali, Z., Parisi, M., Shapovalov, Y., O’Banion, M. K., et al. (2008). ALS-causing SOD1 mutants generate vascular changes prior to motor neuron degeneration. Nat. Neurosci. 11, 420-422. doi: 10.1038/ nn2073

Zhou, Y., and Nathans, J. (2014). Gpr124 controls CNS angiogenesis and bloodbrain barrier integrity by promoting ligand-specific canonical wnt signaling. Dev. Cell 31, 248-256. doi: 10.1016/j.devcel.2014.08.018

ZhuGe, Q., Zhong, M., Zheng, W., Yang, G. Y., Mao, X., Xie, L., et al. (2009). Notch-1 signalling is activated in brain arteriovenous malformations in humans. Brain 132, 3231-3241. doi: 10.1093/brain/awp246

Conflict of Interest: The authors declare that the research was conducted in the absence of any commercial or financial relationships that could be construed as a potential conflict of interest.

Copyright (c) 2020 Vieira, Shah and Ruiz de Almodovar. This is an open-access article distributed under the terms of the Creative Commons Attribution License (CC BY). The use, distribution or reproduction in other forums is permitted, provided the original author(s) and the copyright owner(s) are credited and that the original publication in this journal is cited, in accordance with accepted academic practice. No use, distribution or reproduction is permitted which does not comply with these terms. 